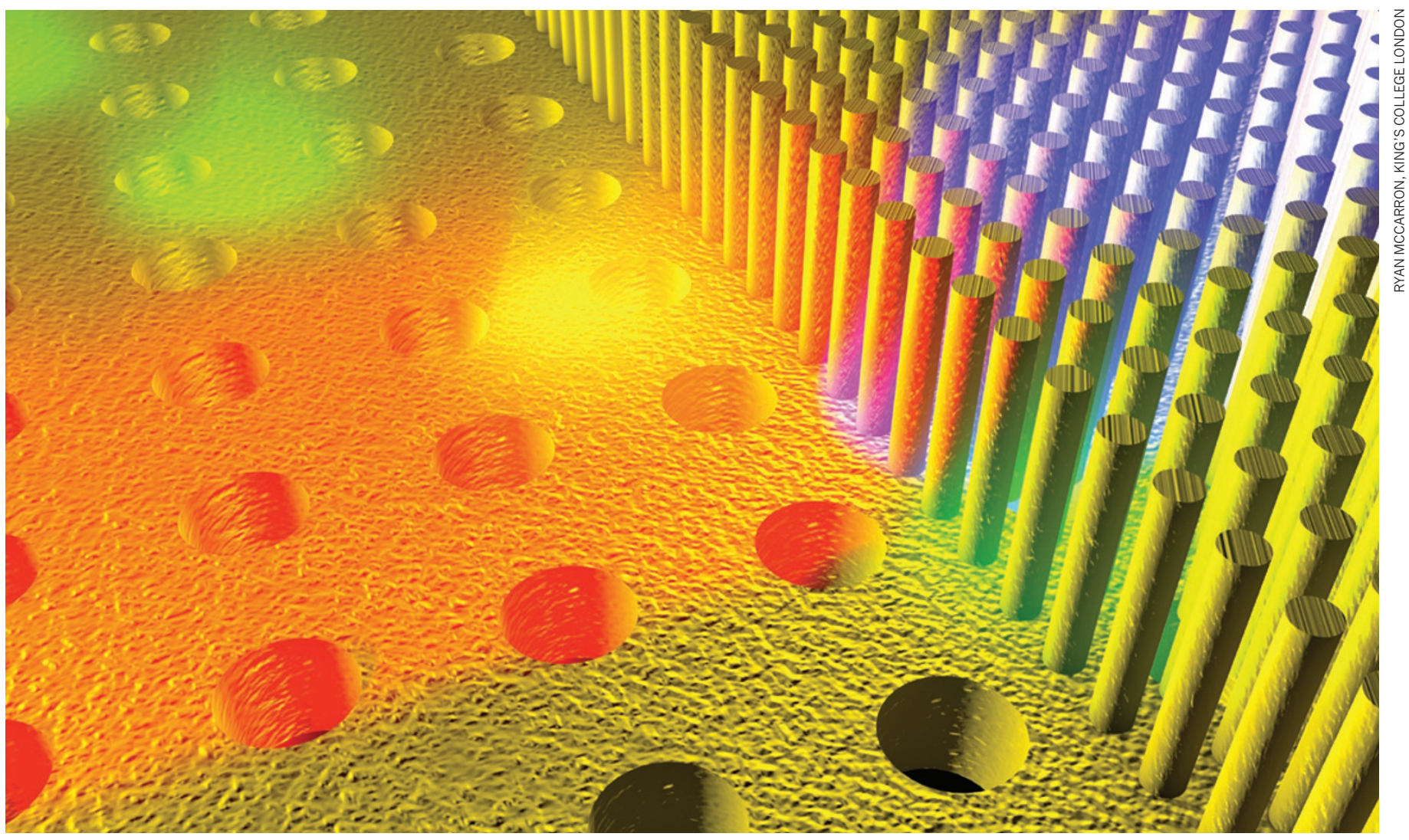

Because of their interaction with light, called plasmonic resonance, gold nanoparticles can be used to make effective sensors and efficient solar cells.

\title{
Trick of the light
}

\section{Invisibly small particles of gold can be used to manipulate the properties of light.}

\section{BY NEIL SAVAGE}

A very special piece of glass can be found at the British Museum in London. Forged into a chalice in the fourth century, it depicts the mythological King Lycurgus being trapped by a vine after he attacked the god Dionysus. What sets this cup apart from other ancient artefacts is the way it interacts with light - in ambient light it is jade in colour, but when light shines through it, it glows bright red.

This striking phenomenon arises from tiny beads of gold and silver, about 70 nanometres in diameter, embedded in the glass. At these dimensions, a phenomenon called surface plasmon resonance takes effect: light striking the metal's surface resonates with the metal's electrons, redirecting colours of light in unusual ways.

Gold has a long history in electronics and optics, from corrosion-resistant electrical contacts to sunlight filters in astronauts' helmets. Research now shows how gold at the nanometre scale can be used to exploit light: a sprinkling of gold dust can improve solar cell technology or make more sensitive biodetectors.

"In ancient times, people already knew how to use gold to obtain fantastic and predictable optical effects," says Cesare Umeton, a physicist at the University of Calabria, Italy, who is working on decidedly more modern applications of plasmonics. "You have to use gold and other particles to have particular effects in optics."

Although plasmonic effects occur in a variety of metals, scientists prefer using gold. "For some applications, other materials might have better properties," says physicist Anatoly Zayats at King's College London. But when researchers consider all the properties together, gold usually wins. It works with visible and nearinfrared light - wavelengths important for several growing technologies, including photovoltaics, optical communications, biosensors and display technology. It's also malleable in a molecular sense: groups of functional molecules (ligands) can be attached easily. And in applications in which silver or aluminium would be rendered useless in a matter of weeks by oxidization, gold still works.
Plasmons are electromagnetic oscillations that occur at the surface of a metal nanoparticle when it is struck by a photon and is in contact with a dielectric material - an insulator, such as glass, that can store an electrostatic charge. Electrons oscillate in resonance with the frequency of the incoming light, amplifying the light at a particular wavelength to make a colour brighter; which colour depends on the metal in question, the size of the nanoparticle, and the nature of the insulator. Nanoparticles can also act as antennae, collecting light energy and transmitting it to another material.

\section{CAPTURING SUNLIGHT}

The ability of metal nanoparticles to act like an antenna is helping Yang Yang, a materials physicist at the University of California, Los Angeles, to improve the efficiency of polymer solar cells - their efficiency at converting sunlight to electricity is counted in single digits, compared with almost $20 \%$ efficiency achieved with conventional silicon solar cells. The polymer cells, however, are lighter, simpler and potentially much cheaper. 
Yang works on tandem solar cells in which thin films of polymer are stacked on top of one another. Because each layer is made of an organic semiconductor tuned to a different wavelength, the design promises to capture a broader spectrum of sunlight. To make the cells more sensitive, Yang mixes gold nanoparticles about $500-600 \mathrm{~nm}$ in diameter into a common conductive polymer called PEDOT, which serves as a connector between the layers. The gold captures light and delivers it to the next layer of semiconductor. "You can think of it as an amplifier," Yang says. Using the gold nanoparticles, Yang increased the efficiency of the tandem solar cell from $5.22 \%$ to $6.24 \%$ (ref. 1).

With even these modest rates of efficiency, polymer solar cells could become a valuable power source because they can be coated onto $\sum$ windows without the expense or weight considerations of a rooftop silicon installation. "If we can turn half the windows in New York City into solar cells with 5-10\% efficiency, I think we can really do something for the solar industry," Yang says. He estimates that in some locations, solar cells applied to the windows of one 30 -storey building could provide enough electricity to meet the needs of 25 households ${ }^{1}$.

Gold could also improve solar cells in a different way. Zayats makes slits $100 \mathrm{~nm}$ wide in thin gold films, with varied spacing between the slits ${ }^{2}$. This produces a plasmonic rainbow effect, where different frequencies of plasmonic waves arise on different parts of the gold grating, interacting with various wavelengths of light. The device acts as a broadband antenna that could, for example, enable a solar cell to capture a wider range of wavelengths. The gratings work equally well regardless of the angle of light, so there is no need to mechanically redirect the panels towards the Sun.

But Zayats thinks his plasmonic rainbow could be more useful in sensors. Many organic molecules can be identified by the infrared light they absorb, but existing detectors don't work well at those wavelengths. A plasmonic grating that focuses light onto a sensor would make them much more sensitive.

Another type of highly effective sensor to detect drugs or explosives could be based on a different way of amplifying light, called surface-enhanced Raman spectroscopy, according to chemists Alexei Kornyshev and Joshua Edel of Imperial College London. Their device is simple: they took gold particles about $43 \mathrm{~nm}$ in diameter with molecules of citric acid attached, and mixed these nanoparticles with water, sodium chloride and an organic solvent ${ }^{3}$. The nanoparticles lined up in a thin film at the boundary between the water and the organic solvent. By changing the sodium chloride concentration, they can control the spacing between the nanoparticles: when spaced close together (but not touching), the plasmonic effect creates a 'hot spot' between the nanoparticles that amplifies light by 10-12 orders of magnitude, Edel says.
To test a sample, they mix it in and shine a laser beam on the tube, then analyse the outgoing light with a spectrograph, which splits light into its composite frequencies. A molecule will bind either the citric acid or the gold itself and cause a distinctive spectral shift, amplified by the hot spot. In just a few seconds the sensor can detect a drug, explosive or pollutant present in quantities of just a dozen molecules, according to Edel.

\section{META METAL}

Gold can be used to build materials with properties that seem to defy common sense. One such metamaterial attracts light from an area larger than itself. The material, built at Duke University in Durham, North Carolina,

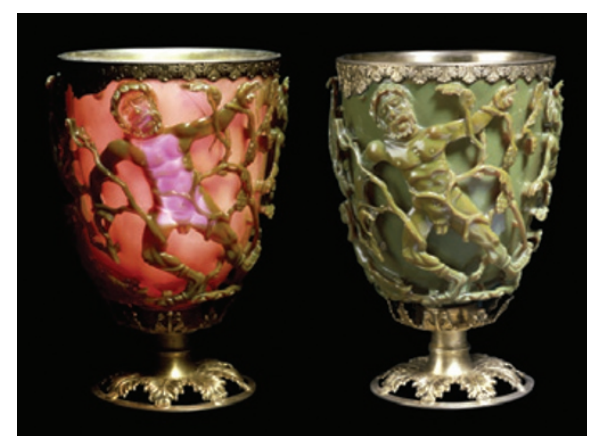

Glowing for gold: metal nanoparticles turn the Lycurgus Cup red when light is shone through it.

consists of a thin film of gold covered with a transparent polymer, with silver nanocubes scattered over the polymer surface at a density of up to 30 million cubes per square millimetre. With the right spacing between the underlying metal and the silver cubes, plasmon resonance alters the electromagnetic properties of the thin film in an area 30 times as large as the cube itself, cancelling out its ability to reflect light ${ }^{4}$. So carefully covering about $3 \%$ of a surface could lead to the absorption of all the light striking that surface. "All the light gets trapped under the cube," explains Antoine Moreau, a nanophotonics researcher at Blaise Pascal University in Clermont-Ferrand, France, who worked with the Duke team. Such total absorption might be useful for turning light into heat and then generating electricity in thermophotovoltaics. Engineering the material to control which wavelength it captures could lead to a nano-ink for security printing on currency or seals of authenticity.

The first three-dimensional metamaterials that work with infrared or visible light were created in 2008, and they are still expensive and time consuming to produce. But the Duke process relies on self-assembly, selecting the chemical and physical properties of the components so they form the desired structure, just as Edel's sensors do. In fact, much of the appeal of recent work with nanoparticles is that they rely on potentially cheap 'bottomup' assembly.

\section{SHRINKING ELECTRONICS}

Nanoparticles might also serve as the building blocks for nanoelectronics. Umeton, for instance, says that he can build a nanoscale resistance-inductance-capacitance (RLC) circuit, an important component in radio signal filtering and tuning. He does this by mixing gold nanoparticles into liquid crystals in a diffraction grating, which spreads out the wavelengths of light like a prism. Choosing a liquid crystal material with a particular refractive index lets him select which wavelengths to work with, and applying an electric field lets him switch the index between two states ${ }^{5}$.

"You can use this in any kind of nanoelectric circuit," Umeton says. "You have the possibility to transfer to the nanoscale the know-how you have for electronics."

The gold-in-liquid-crystal technology could lead to displays built on flexible substrates such as plastic, suggests his colleague at the University of Calabria, physicist Roberto Caputo. "In principle, you would illuminate it with white light and get all the colours you need by using the plasmonic response," he says. With no colour filters, lower energy consumption and the ability to bend, such screens could be wrapped around telephone poles or sewn into clothing.

Gold-based plasmonics could reach inside computers as well. Engineers would like to use light to route data between processors on computer chips, because it can carry much more data quicker than an electrical signal. But as Zayats points out, silicon waveguides to channel the light have diameters of a few hundred nanometres, compared with the tens of nanometres used in electrical connections. Plasmonics offer a solution: they can squeeze light into spaces much smaller than the light's wavelength. Several groups, from Stanford University in California to the University of Twente in Enschede, the Netherlands, are currently developing plasmonic waveguides, and Zayats says they're almost ready for production.

Caputo points to another way gold could speed up computers. Liquid crystals can serve as the basis of microlasers. Caputo says selecting the concentration of gold nanoparticles within these crystals would allow engineers to choose different colours of laser light. A laser array on a chip could provide high-capacity interconnects - yet one more golden opportunity for those tiny flecks of yellow metal to have an outsized impact on technology.

Neil Savage is a science and technology writer based in Lowell, Massachusetts.

1. Yang, J. et al. ACS Nano 5, 6210-6217 (2011).

2. Bouillard, J.-S. et al. Sci. Rep. 2, 829 (2012).

3. Cecchini, M. P. et al. Nature Mat. 12, 165-171 (2012).

4. Moreau, A. et al. Nature 492, 86-89 (2012).

5. Caputo, R. et al. Asia Commmunications and Photonics Conference paper PAF4D.8 (Optical Society of America, 2012). 